\title{
Performance and Return to Sport After Hand, Wrist, and Forearm Fractures in the National Hockey League
}

\author{
Matthew J. Gotlin, M.D., Shobhit V. Minhas, M.D., Daniel B. Buchalter, M.D., \\ Oren I. Feder, M.D., Michael J. Alaia, M.D., and Laith M. Jazrawi, M.D.
}

\begin{abstract}
Purpose: To examine finger, thumb, hand, wrist, and forearm fractures in the National Hockey League (NHL) and determine: (1) basic demographic data, (2) return to sport (RTS) rates, (3) performance after RTS, and (4) the difference in RTS between players treated operatively versus conservatively. Methods: NHL players with finger, thumb, hand, wrist, and forearm fractures between the 1995-1996 and 2014-2015 seasons were identified through team injury reports and archives on public record. Player demographics, RTS rate, games played per season, and performance score for each player were recorded and compared between the preinjury season and one season following injury. Results: A total of 247 total NHL players with hand, wrist, and forearm fractures were identified, consisting of 30.8\% finger, 38.5\% hand, $13.8 \%$ thumb, $14.6 \%$ wrist, and $2.4 \%$ forearm fractures. Defenseman comprised the majority of players $(40.1 \%)$. The overall RTS rate was $98.0 \%$, with no significant difference between players with surgery or between injury location groups. In total, 52 players $(21.1 \%)$ underwent surgery with no significant correlation of surgery rates based on fracture location. The mean number of missed games was $13.8 \pm 9.9$, with players sustaining wrist and forearm fractures missing the largest number of games $(21.6 \pm 17.7$ and $22.8 \pm 7.5$ games missed, respectively). There was no significant change in games played or performance scores 1 year after injury for players with any of the fracture types compared with baseline preoperative games played and performance. Conclusions: NHL players have a high RTS rate following hand, wrist, and forearm fractures. Players were able to return to preinjury performance within l year, regardless of treatment or type of fracture. Level of Evidence: Level IV, case series.
\end{abstract}

$\mathbf{P}$ rofessional hockey is a high-speed collision sport with a reported injury rate of 14.2 to 78.4 injuries per 1000 player game hours. ${ }^{1,2}$ Players ice skate at speed up to $30 \mathrm{mph}$ and propel pucks at speeds up to $100 \mathrm{mph}^{3}$ Improvements in helmets and facemasks have significantly diminished the incidence of facial, eye, and dental injuries; however, blunt trauma, specifically collision with opponents, remains the most

From NYU Langone Orthopedic Hospital, Department of Orthopedic Surgery, NYU Langone Health, New York, New York, U.S.A.

The authors report that they have no conflicts of interest in the authorship and publication of this article. Full ICMJE author disclosure forms are available for this article online, as supplementary material.

Received February 6, 2020; accepted May 23, 2020.

Address correspondence to Matthew Gotlin, M.D., NYU Langone Orthopedic Hospital, Department of Orthopedic Surgery, NYU Langone Health, 301 East 17th St., New York, NY 10003.E-mail:matthew.gotlin@nyulangone.org

(C) 2020 THE AUTHORS. Published by Elsevier Inc. on behalf of the Arthroscopy Association of North America. This is an open access article under the CC BY-NC-ND license (http://creativecommons.org/licenses/by-nc-nd/4.0/).

2666-061X/20176

https://doi.org/10.1016/j.asmr.2020.05.013 common cause of injury. ${ }^{3}$ Of the athletes registered in the 2010 Olympics, the risk for sustaining injury was greatest for ice hockey. ${ }^{4}$ To that note, North American Hockey is considered more physical and aggressive compared with that in Europe.

National Hockey League (NHL) players commonly miss games due to injury, which portends significant financial implications in lost salary costs. ${ }^{6}$ Many hockey injuries are well reported in the literature, including concussions, knee ligament ruptures, shoulder separations, femoroacetabular impingement, lumbar disc herniations, and high ankle sprains. ${ }^{2,7-9}$ However, there is a paucity of literature on hand, wrist, and forearm injuries in professional hockey. Makhni et al. ${ }^{10}$ performed a comprehensive review of sports reporting in the medical literature and found that in articles addressing NHL injuries, the greatest proportion of studies addressed the hip/pelvis/upper leg.

The hand, wrist, and forearm are commons site of ice hockey-related injury, with reported rates in the literature from $8 \%$ to $34.4 \% .^{3,11,12}$ Deits et al. ${ }^{13}$ found that $36 \%$ of ice hockey injuries presenting to U.S. 
emergency departments were related to upperextremity injuries. There is currently no reported incidence of upper-extremity fractures in the NHL. Ice hockey players, unlike other sportsmen, have a unique desire to return to sports, even after injuries that sideline other athletes for long periods of time. ${ }^{14}$ With the NHL's increasing popularity and high incidence of injury, further research is needed to understand the injuries associated with the sport and to develop performance based outcome measure to guide safe return to play. ${ }^{15}$

The purpose of this study is to examine finger, thumb, hand, wrist, and forearm fractures in the NHL and determine: (1) basic demographic data, (2) return to sport (RTS) rates, (3) performance after RTS, and (4) the difference in RTS between players treated operatively versus conservatively. We hypothesized that NHL players would demonstrate high RTS rates regardless of fracture type and would be able to perform at preinjury level. We also predicted that operative or conservative treatment would not affect RTS or performance.

\section{Methods}

\section{Data Source}

By using a previous published methodology, we identified NHL players treated either surgically or conservatively for forearm (radius and/or ulnar shaft), wrist, hand, thumb, and finger fractures between the 1995-1996 and 2014-2015 seasons through team injury reports, player profiles, and press releases. ${ }^{7,16-19}$ Inclusion criteria included those players who were placed on the injured list, confirmed by team transaction reports, cross-checked from official NHL team websites, and corroborated by at least 2 independent public sources. Of note, players with concomitant injuries requiring surgery (to minimize confounding variables) and players who did not have RTS (and hence did not have postinjury season statistics) were excluded from performance variable analyses but were still included in RTS analyses. We chose to exclude fracture of the upper extremity proximal to the forearm due to the lack of detailed reporting of these injury types in the available sources.

\section{Player and Performance Variables}

Player variables included body mass index, player position, and shooting hand. Fracture location (forearm, wrist, hand, thumb, and finger) as well as surgical versus nonoperative intervention also was recorded. Performance variables included games played, number of RTS, defined as a return to the active roster for at least 1 professional regular-season game, and a performance score modified for hockey as previously published (Table 1). ${ }^{9}$
Table 1. Performance Score Formula for a Given Position

\begin{tabular}{cc}
\hline Center/wing & {$[(3.0 \times$ even-strength goals $)+(2.0 \times$ power play } \\
& goals $)+(4.0 \times$ short-handed goals $)+(4.0 \times$ \\
& game-winning goals $)+(2.0 \times$ assists $)+($ plus $/$ \\
& minus $)-(0.25 \times$ penalty in minutes $)-(0.33 \times$ \\
& shot on goal $)] /$ \\
& Games Played \\
Defenseman & {$[(5.0 \times$ even strength goals $)+(4.0 \times$ power play } \\
& goals $)+(6.0 \times$ short-handed goals $)+(5.0 \times$ \\
& game-winning goals $)+(3.0 \times$ assists $)+($ plus $/$ \\
& minus $)-(0.25 \times$ penalty in minutes $)-(0.33 \times$ \\
& shots on goal $)] /$ \\
& Games Played \\
& {$[(0.7 \times$ wins $)+(0.2 \times$ ties plus overtime losses $)+$} \\
Goalie & shutouts $+(0.17 \times$ saves $)-(0.25 \times$ losses $)-$ \\
& $(1.23 \times$ goals against $)[/$ \\
& Games Played
\end{tabular}

NOTE. These are the equations used to calculate the performance score for specific positions.

\section{Statistical Analysis}

IBM SPSS, Version 23 (IBM Corp., Armonk, NY) was used to perform all descriptive and comparative analyses. RTS rates between operative and nonoperative intervention were compared by using the Fisher exact test. Mean missed games were compared through independent $t$ tests and the Levene test of equality. Postinjury season and preinjury season games played and performance scores were compared using paired $t$ tests. For all analyses, a $P$ value of less than or equal to .05 was deemed statistically significant.

\section{Results}

A total of 247 total NHL players with hand, wrist, and forearm were identified, consisting of $30.8 \%$ finger, $38.5 \%$ hand, $13.8 \%$ thumb, $14.6 \%$ wrist, and $2.4 \%$ forearm fractures (Table 2). Defenseman comprised the majority of players $(40.1 \%)$ (Table 2). The overall RTS rate was $98.0 \%$, with no significant difference between players with surgery or between injury location groups. The players who did not experience RTS and the

Table 2. Demographics of Players

\begin{tabular}{lrr}
\hline Player/Injury Characteristic & $\mathrm{n}$ & $\%$ \\
\hline BMI & & $26.7 \pm 1.4$ \\
Shoots with left hand & 49 & $60.7 \%$ \\
Center & 99 & $19.8 \%$ \\
Defenseman & 90 & $40.1 \%$ \\
Wing & 9 & $36.4 \%$ \\
Goalies & 76 & $3.6 \%$ \\
Finger fracture & 34 & $30.8 \%$ \\
Thumb fracture & 95 & $13.8 \%$ \\
Hand fracture & 36 & $38.5 \%$ \\
Wrist fracture & 6 & $14.6 \%$ \\
Forearm fracture & $2.4 \%$ \\
\hline NOTE. The table contains demographic data relating to player and \\
injury characteristics. & & \\
BMI, body mass index. & &
\end{tabular}


characteristics of their injuries are listed in Table 3. In total, 52 players $(21.1 \%)$ underwent surgery with no significant correlation of surgery rates based on fracture location. The mean number of missed games was 13.8 \pm 9.9 , with players sustaining wrist and forearm fractures missing the largest number of games $(21.6 \pm 17.7$ and $22.8 \pm 7.5$ games missed, respectively) (Table 4). Other than hand fractures, all other fracture locations resulted in a statistically greater mean number of games missed with surgery compared with nonoperative treatment (Table 5). Of note, due to the few number of forearm fractures, missed games were unable to be statistically compared. There was no significant change in games played or performance scores 1 year after injury for players with any of the fracture types compared with baseline preoperative games played and performance (Figs 1 and 2).

\section{Discussion}

Our results demonstrated that the RTS rate after sustaining a hand, wrist, or forearm fracture is extremely high. The RTS rate did not differ between fracture location or between players who had surgery versus those who were treated conservatively. We also found that players were able to return to preinjury performance after sustaining any hand, wrist, or forearm fracture within 1 year. As previously mentioned, the authors could find no studies to date that have looked at the RTS or the performance after RTS in the NHL after hand, wrist, and forearm fractures. However, when compared with studies looking at hand, wrist, and forearm fractures in other professional sports, hockey players miss fewer games and have RTS at greater rates.

Morse et al. ${ }^{20}$ looked at metacarpal and phalangeal fracture in National Basketball (NBA) players. Compared with NHL players, NBA players with metacarpal and phalangeal fractures missed on average more games (16.3 games vs 14.2 games, and 11.4 games vs 10.2 games, respectively.) Both the NHL and NBA average about 3 games per week during their regular season; therefore, the data are comparable. Our study parallels other research that has determined high RTS and maintenance of preinjury performance after

Table 3. Players Who Did Not Return to Sport

\begin{tabular}{llll}
\hline Players & Injury Type & Position & Surgery Required \\
\hline 1 & Hand fracture & Center & Yes \\
2 & Hand fracture & Defenseman & No \\
3 & Hand fracture & Goalie & No \\
4 & Index finger & Right wing & No \\
& fracture & Right wing & No \\
5 & Thumb fracture & Rid not return to \\
\hline \multicolumn{2}{l}{ NOTE. Characteristics of injuries in those who did nort are shown. }
\end{tabular}

Table 4. Mean Number of Missed Games by Injury

\begin{tabular}{lc}
\hline Fracture Location & Mean Number of Missed Games \\
\hline Finger & $10.2 \pm 5.7$ \\
Hand & $14.1 \pm 6.9$ \\
Thumb & $10.4 \pm 6.4$ \\
Wrist & $21.6 \pm 17.7$ \\
Forearm & $22.8 \pm 7.5$ \\
Total & $13.8 \pm 9.9$ \\
\hline
\end{tabular}

NOTE. The mean number of games missed are listed for different fracture locations.

hand, wrist, and forearm fractures. Sochacki et al. ${ }^{21}$ found a high rate of RTS and maintained postoperative performance following forearm open reduction and internal fixation in NHL players. Guss et al. ${ }^{22}$ found no significant change in performance and high RTS in NBA players who sustained metacarpal fractures.

Our study also found that hand fractures were the most common. Previous reports have shown that the majority of injuries are caused by collision with an opponent or with the boards ${ }^{3}$; however, the implications of the high rate of hand fractures imply less common methods of injury, such as being struck by the puck or hockey stick, as well as fist fights. Defenseman were the most commonly injured position (40.1\%), which is consistent with the reported rate of overall orthopaedic injuries in defensemen in previous studies. ${ }^{11}$ Goalies were the least likely to sustain hand, wrist, or forearm fracture, presumably due to their massive upper-extremity protective gear. Furthermore, RTS rates were high, with no significant impact of surgery or type of fracture. However, players who were treated operatively missed more games on average than those players who were treated conservatively. This may be secondary to more complex fractures requiring surgical intervention and less severe fractures being amenable to nonoperative management.

Table 5. Mean Missed Games Based on Injury and Treatment

\begin{tabular}{llrc}
\hline Fracture Location & Intervention & $\mathrm{n}$ & $P$ Value \\
\hline Finger & Operative & $14.0 \pm 5.2$ & $0.017^{*}$ \\
& Nonoperative & $9.6 \pm 5.3$ & \\
Hand & Operative & $16.2 \pm 6.6$ & 0.064 \\
& Nonoperative & $12.9 \pm 6.7$ & \\
Thumb & Operative & $17.8 \pm 6.1$ & $0.023^{*}$ \\
\multirow{2}{*}{ Wrist } & Nonoperative & $8.6 \pm 5.1$ & \\
& Operative & $35.4 \pm 24.4$ & $0.002^{*}$ \\
Forearm & Nonoperative & $15.7 \pm 8.7$ & \\
& Operative & N/A & N/A \\
& Nonoperative & N/A & N/A \\
\hline
\end{tabular}

NOTE. The number of mean games missed are listed for each fracture location based on whether their fracture was treated operatively or nonoperatively.

N/A, not available.

*Represents values that are statistically significant $(P<0.05)$. 


\section{Change in Games Played}

25.0

20.0

15.0

10.0

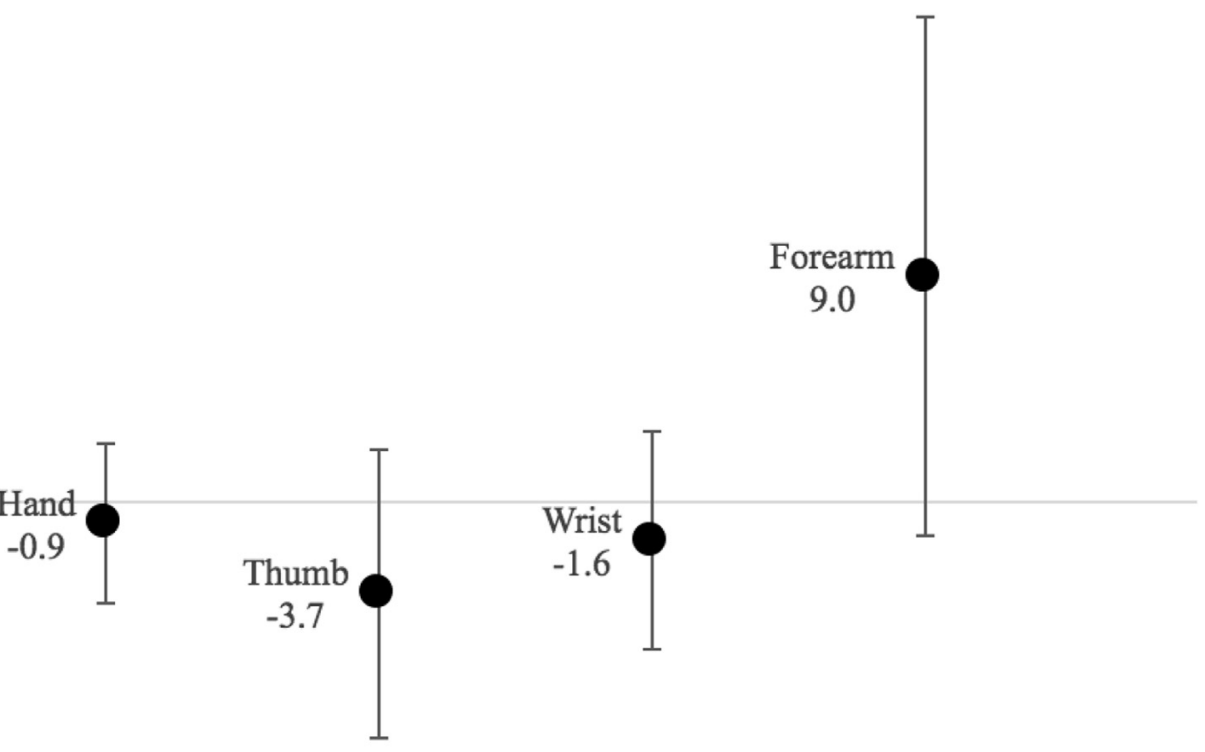

$-10.0$

5.0

0.0

$-5.0$

Finger
0.4

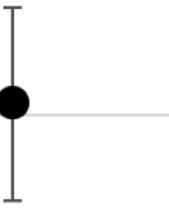

$\underset{-0.9}{\text { Hand }}$

$-15.0$

Fig 1. Change in games played the season following injury. This figure depicts the change in games played for different fracture locations. Data points below the zero line demonstrate a reduction in games played the season after the injury, whereas data points above the zero line demonstrate an increase in games played the season after injury. None of the data points were statistically significant.

Unfortunately, the radiographs, operative reports, or details about fracture severity were not available, so recommendations about specific fracture management cannot be deduced. Treatment decisions should be based on individual fracture characteristics and should not be influenced by sooner return to play seen in the nonoperative group. In addition, players with wrist and forearm injuries missed the most games, regardless of operative versus conservative management. This study sheds light on the impact of hand, wrist, or forearm fractures in NHL players, and suggests that players can predictably experience RTS at a high-performance level.

\section{Limitations}

This study is not without its limitations. Players were identified using publicly available internet sources, which is subject to observer bias. Some injuries may have been missed due to minimal media coverage of lesser-profile players or anticipated media and/or public scrutiny. Nevertheless, this method of subject selection has been used in multiple high-evidence level studies in sports medicine journals. ${ }^{7,16-19}$ Concomitant injuries can impact performance scores and games played. We excluded players who had reported concomitant injuries requiring surgery during the time period in our study. However, we included players with concomitant injuries that did not require surgery. This could influence our performance analysis and should be considered when looking at our reported results on performance.

In addition, severity of fracture and management strategies were not known and presumably not consistent among all players. No patient-reported or clinician-reported outcomes were available. Players were excluded from the study if they were transferred to the minor leagues or if they were a rookie during their injury. These missed players could have had potential to alter our RTS and performance data. Furthermore, elbow, humeral shaft, and proximal humerus fractures were not included in this analysis because reporting of these injuries in the sources available were lacking significant detail. Fractures above the elbow were commonly reported as "arm fracture" or "upper arm injury." In addition, only fractures that resulted in missed games that were 


\section{Change in Performance Score}

1.0

0.8

0.6

0.4

0.2

0.0

$-0.2$

Finger
-0.09

2

$-0.4$

$-0.6$

$-0.8$

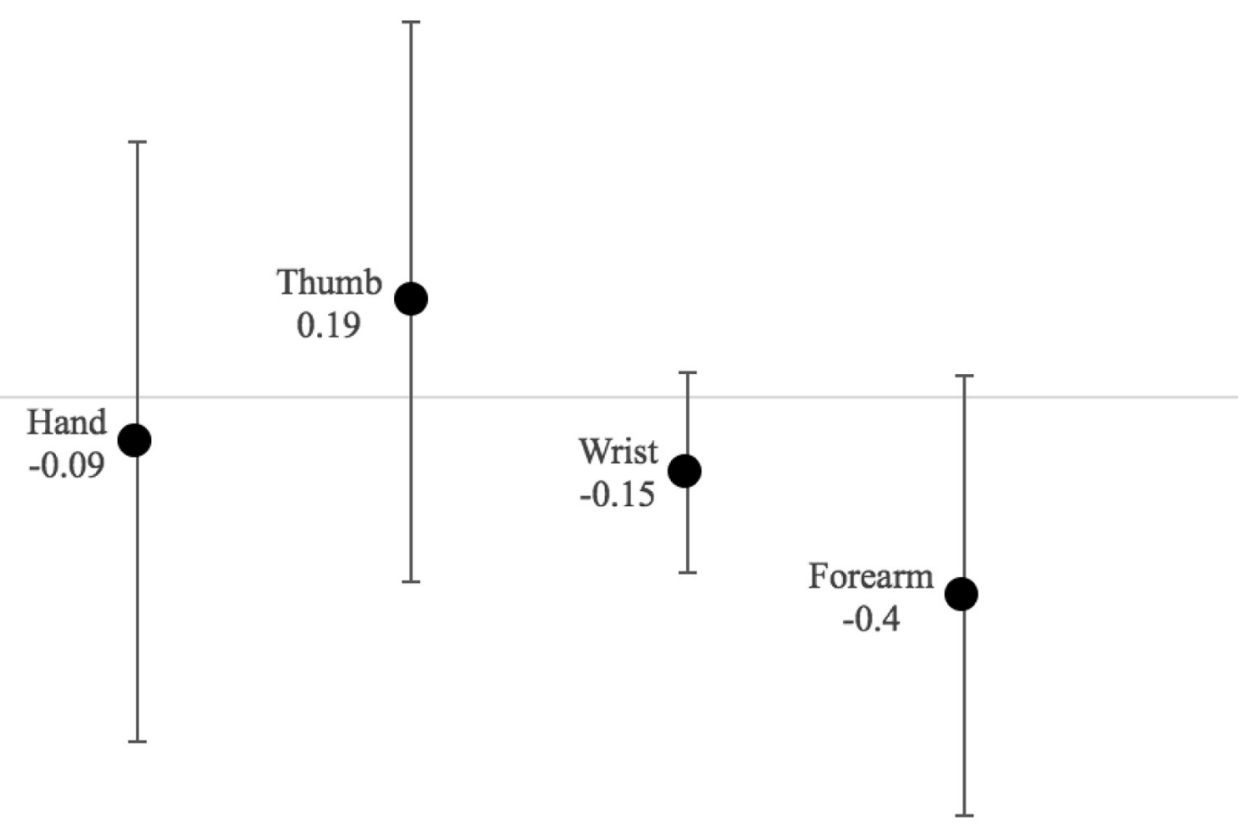

$-1.0$

Fig 2. Change in performance score the season following injury. This figure depicts the change in performance for different fracture locations. Data points below the zero line demonstrate a reduction in performance the season after the injury, whereas data points above the zero line demonstrate an increase in performance the season after injury. None of the data points were statistically significant.

publicly reported were included, and there may be fractures that resulted in no games missed. Lastly, intangibles such as coach and front office decision making, teammate motivation, and team leadership cannot be assessed.

\section{Conclusions}

NHL players have a high RTS rate following hand, wrist, and forearm fractures. Players were able to return to preinjury performance within 1 year, regardless of treatment or type of fracture.

\section{References}

1. Lorentzon R, Wedren H, Pietila T. Incidence, nature, and causes of ice hockey injuries. A three-year prospective study of a Swedish elite ice hockey team. Am J Sports Med $1988 ; 16: 392-396$.

2. Longstaffe R, Leiter J, MacDonald P. Anterior cruciate ligament injuries in the national hockey league: Epidemiology and performance impact. Clin J Sport Med 2020;30:224-230.
3. Flik K, Lyman S, Marx RG. American collegiate men's ice hockey: an analysis of injuries. Am J Sports Med 2005;33: 183-187.

4. Engebretsen L, Steffen K, Alonso JM, et al. Sports injuries and illnesses during the Winter Olympic Games 2010. Br J Sports Med 2010;44:772-780.

5. Biasca N, Simmen HP, Bartolozzi AR, Trentz O. Review of typical ice hockey injuries. Survey of the North American NHL and Hockey Canada versus European leagues. Unfallchirurg 1995;98:283-288.

6. Donaldson L, Li B, Cusimano MD. Economic burden of time lost due to injury in NHL hockey players. Inj Prev 2014;20:347-349.

7. Erickson BJ, Harris JD, Cole BJ, et al. Performance and return to sport after anterior cruciate ligament reconstruction in National Hockey League players. Orthop $J$ Sports Med 2014;2:2325967114548831.

8. Kuhn AW, Zuckerman SL, Totten D, Solomon GS. Performance and style of play after returning from concussion in the National Hockey League. Am J Sports Med 2016;44:2152-2157.

9. Schroeder GD, McCarthy KJ, Micev AJ, Terry MA, Hsu WK. Performance-based outcomes after nonoperative treatment, discectomy, and/or fusion for a lumbar 
disc herniation in National Hockey League athletes. Am J Sports Med 2013;41:2604-2608.

10. Makhni EC, Buza JA, Byram I, Ahmad CS. Sports reporting: A comprehensive review of the medical literature regarding North American professional sports. Phys Sportsmed 2014;42:154-162.

11. Agel J, Dompier TP, Dick R, Marshall SW. Descriptive epidemiology of collegiate men's ice hockey injuries: National Collegiate Athletic Association Injury Surveillance System, 1988-1989 through 2003-2004. J Athl Train 2007;42:241-248.

12. Mosenthal W, Kim M, Holzshu R, Hanypsiak B, Athiviraham A. Common Ice hockey injuries and treatment: A current concepts review. Curr Sports Med Rep 2017;16:357-362.

13. Deits J, Yard EE, Collins CL, Fields SK, Comstock RD. Patients with ice hockey injuries presenting to US emergency departments, 1990-2006. J Athl Train 2010;45: 467-474.

14. LaPrade RF, Wijdicks CA, Griffith CJ. Division I intercollegiate ice hockey team coverage. Br J Sports Med 2009;43: 1000-1005.

15. Laprade RF, Surowiec RK, Sochanska AN, et al. Epidemiology, identification, treatment and return to play of musculoskeletal-based ice hockey injuries. Br J Sports Med 2014:48:4-10.
16. Amin NH, Old AB, Tabb LP, Garg R, Toossi N, Cerynik DL. Performance outcomes after repair of complete Achilles tendon ruptures in national basketball association players. Am J Sports Med 2013;41:1864-1868.

17. Cerynik DL, Lewullis GE, Joves BC, Palmer MP, Tom JA. Outcomes of microfracture in professional basketball players. Knee Surg Sports Traumatol Arthrosc 2009;17:1 135-1139.

18. Erickson BJ, Harris JD, Cvetanovich GL, et al. Performance and return to sport after anterior cruciate ligament reconstruction in male Major League Soccer players. Orthop J Sports Med 2013;1:2325967113497189.

19. Lohmander LS, Englund PM, Dahl LL, Roos EM. The long-term consequence of anterior cruciate ligament and meniscus injuries: osteoarthritis. Am J Sports Med 2007;35: 1756-1769.

20. Morse KW, Hearns KA, Carlson MG. Return to play after forearm and hand injuries in the National Basketball Association. Orthop J Sports Med 2017;5:2325967117690002.

21. Sochacki KR, Jack RA 2nd, Hirase T, et al. Performance and return to sport after forearm fracture open reduction and internal fixation in National Football League players. Hand (N Y) 2017:1558944717726137.

22. Guss MS, Begly JP, Ramme AJ, Hinds RM, Karia RJ, Capo JT. Performance outcomes after metacarpal fractures in National Basketball Association players. Hand ( $N$ Y) 2016;11:427-432. 